\title{
A genome-wide sequence-structure analysis suggests aggregation gatekeepers constitute an evolutionary constrained functional class
}

Greet De Baets ${ }^{1,2,3}$, Joost Van Durme ${ }^{1,2,3}$, Frederic Rousseau ${ }^{1,2, *}$ \& Joost Schymkowitz ${ }^{1,2, *}$

1 - VIB Switch Laboratory, Flanders Institute for Biotechnology (VIB), Leuven, Belgium

2 - Switch Laboratory, Department of Cellular and Molecular Medicine, University of Leuven, Herestraat 49, 3000 Leuven, Belgium

3 - Vrije Universiteit Brussel, Pleinlaan 2, 1050 Brussels, Belgium

To whom correspondence should be addressed: Frederic Rousseau, SWITCH

Laboratory, Department of Molecular Cell Biology, University of Leuven, B-3000

Leuven, Belgium Tel.: +32-16-372572; Fax: +32-16-372751; E- mail:

Frederic.Rousseau@switch.vib-kuleuven.be

or Joost Schymkowitz, SWITCH Laboratory, Department of Molecular Cell Biology, University of Leuven, B-3000 Leuven, Belgium Tel.: +32-16-372573; Fax: (858) 784-2779; E- mail: Joost.Schymkowitz@switch.vib-kuleuven.be 


\begin{abstract}
Protein aggregation is geared by aggregation prone sequences (APRs) that selfassociate by $\beta$-strand interactions. Charged residues and prolines are enriched at the flanks of APRs resulting in decreased aggregation. It is still unclear what drives the overrepresentation of these 'aggregation gatekeepers', i.e. whether their presence results from structural constraints determining protein stability or whether they constitute a bona fide functional class selectively maintained to control protein aggregation. As functional residues are typically conserved regardless of their cost to protein stability we compared sequence conservation and thermodynamic cost of these residues in 2659 protein families in E. coli. Across protein families we find gatekeepers to be under strong selective conservation while at the same time representing a significant thermodynamic cost to protein structure. This finding supports the notion that aggregation gatekeepers are not structurally determined but evolutionary selected to control protein aggregation.
\end{abstract}




\section{Keywords}

Protein aggregation, thermodynamic stability, gatekeepers, evolution 


\section{Introduction}

Protein aggregation is mediated by short aggregation prone regions (APRs) within protein sequences that can self-assemble by intermolecular $\beta$-strand interactions to form aggregates of misfolded proteins ${ }^{1 ; 2}$. Whereas APRs are generally buried in the hydrophobic core of native globular proteins thereby precluding aggregation, they are solvent exposed and prone to aggregate in situations of physiological stress or during protein translation, trafficking or degradation requiring tight regulation by molecular chaperones ${ }^{3}$.

Proteome-wide analysis of the aggregation propensity of 28 organisms revealed that the flanks of APRs are enriched in charged amino acids and prolines ${ }^{4}$. This observation was further confirmed in other studies $5 ; 6 ; 7 ; 8 ; 9 ; 10$. These residues, termed aggregation gatekeepers, oppose aggregation by introducing charge repulsion $(\mathrm{R}, \mathrm{K}, \mathrm{D}$ and $\mathrm{E})$, unfavorable entropic contribution by side chain immobilization (especially $\mathrm{K} \& \mathrm{R}$ ), or in the case of proline main-chain entropic destabilization of the beta-strand conformation of aggregates ${ }^{11 ; 12 ; 13}$.

In addition to their structural effect as aggregation breakers, gatekeeper residues also appear to contribute to chaperone binding in E.coli $4 ; 8 ; 14 ; 15 ; 16 ; 17$. Finally, gatekeepers also seem to influence protein abundance in E. coli by modifying protein synthesis and degradation rates ${ }^{18}$. Interestingly, the frequency of gatekeeper occurrence is significantly higher in groups of proteins with an essential cellular function $^{7 ; 19 ; 20}$, further supporting their role in maintaining proteostasis. In keeping with this notion, mutations that alter gatekeeper residues occur much more frequently in disease-associated mutations than in polymorphisms ${ }^{8}$.

Despite the wealth of data showing that aggregation gatekeepers affect protein solubility, chaperone binding and the proteostatic regulation of proteins, it is still unclear whether the enrichment of gatekeepers at flanks of APR is a selective process resulting from functional constraints or whether gatekeeper enrichment is a secondary effect subordinate to protein structural constraints. It is generally assumed that the specific placement of these aggregation-opposing residues at the flanks of APRs results from the physical impossibility to accommodate charged residues and prolines in the hydrophobic core without disrupting globular structure ${ }^{10 ; 21}$ and thus as a corollary that flank positioning of gatekeepers represent thermodynamically neutral or favorable positions compatible with protein structure. On the other hand, bona fide 
functional residues are not optimized for protein stability and are therefore often found to be destabilizing to protein structure despite their high degree of conservation 22; 23; 24 . In order to distinguish these two scenarios we here compared the sequence conservation of gatekeepers with the thermodynamic cost of incorporating these residues in the native protein structure of the E. coli genome. For this purpose, we took advantage of a dataset of 34 E. coli genomes for which multiple alignments of orthologs from 2659 genes have been generated ${ }^{25}$. A final set of 1955 genes was extracted from this dataset after removal of all transmembrane proteins, so the analysis below is performed on cytosolic proteins exclusively. 


\section{Results}

APRs can be identified using aggregation prediction algorithms such as TANGO $^{26}$. In the reference E.coli strain K12 MG1655 ${ }^{25}$, we identified 12755 APRs in 1955 genes. As before we find that charged residues and prolines are strongly overrepresented (5-10\% above genome average) at the flanks of APRs (Figure 1A) resulting in a bias towards low aggregation propensity in APRs (Figure 1B) suggesting aggregation is under negative selective pressure ${ }^{4}$. In total we identified 15741 charged residues and prolines at the flanks of APRs: 8542 were found at the Nterminal flank of APRs while 7199 were at the C-terminal flanks (5016 APRs are flanked by gatekeepers at both extremities). As previously observed, we find that the enrichment of proline is stereochemically selective as it is only effective at the Nterminal side ${ }^{18}$.

Aggregation gatekeepers destabilize protein structure. In order to determine whether the enrichment of gatekeepers is subordinate to protein stability, thus whether gatekeepers are thermodynamically neutral or favourable to protein stability, we extracted from our dataset those sequences that have a crystal structure in the PDB ${ }^{27}$ with a resolution better than $4.0 \AA$, extended with homology models for those sequences which had a homologous structure of the same quality with at least $60 \%$ sequence identity. In total, this resulted in a set of 797 out of the 1955 protein families, (coverage of 37\%), consisting of 436 structures and 361 homology models. These structures were used to investigate the thermodynamic cost of incorporating gatekeeper residues in globular proteins using the FoldX force field ${ }^{28}$. A plot of either side chain (Figure 2A) or main chain burial (Figure 2B) of the amino acid residues constituting the APRs in the native structures - binned by TANGO aggregation score confirmed earlier observations ${ }^{10 ; 21}$ that aggregating regions are generally embedded in the hydrophobic core of the protein. Not surprisingly, these residues contribute favorably to the thermodynamic stability of the native protein (median contribution to stability of $-0.95 \mathrm{kcal} / \mathrm{mol}$ ), which is in itself a strong protection against aggregation of native proteins (Figure 2C). Aggregation gatekeepers on the other hand span the entire spectrum of side chain and main chain burial (Figure 3A and 3B, respectively), with the bulk of the gatekeepers displaying partial burial of the side chains (median around $40 \%$ ) and fully buried main chain atoms (median around 85\%). Importantly, 
as a consequence of this still significant burial of charged residues, the majority of gatekeeper residues destabilize protein structure with a median destabilization of 0.8 $\mathrm{kcal} / \mathrm{mol}$ (Figure 3C). In accordance we find that the charged moieties of buried aggregation gatekeepers are almost systematically compensated by salt bridges and hydrogen bonds (Figure 3D \& 3E) to the extent that $15 \%$ of all salt bridges in the 797 structures can be attributed to aggregation gatekeepers. Together these findings demonstrate that contrary to being subordinate to protein structure, the 3414 aggregation gatekeepers flanking 3562 different APRs analyzed here, almost systematically come at a thermodynamic cost to the protein structure in which they are embedded.

Aggregation gatekeepers are selectively conserved. Given the cost to protein stability of gatekeeper motifs we further investigated whether these residues are under selective constraint. In order to do this we investigated gatekeeper polymorphism of the 15741 gatekeepers flanking 12755 APRs in 1955 genes using previously generated multiple alignments of orthologs from 34 E.coli strains ${ }^{25}$. Given the high degree of conservation between multiple genomes of the same species we measured the rate of synonymous and non-synonymous mutations separately as the deviation (in \%) from average genome synonymous and non-synonymous mutation rates respectively ${ }^{29}$. In this manner we compared mutation rates of gatekeepers and APRs and analyzed changes in mutation rates of gatekeepers in function of the aggregation propensity of the associated APR. We found that APR non-synonymous mutation rate does not deviate from genome average non-synonymous mutation rate. Neither did we find an association of the aggregation propensity of APRs with their mutation rate (Figure 4A). In contrast the non-synonymous mutation rate of aggregation gatekeepers is decreased by more than $20 \%$. Importantly, we find a relationship between gatekeeper conservation and the aggregation propensity of the associated APR $\left(R^{2}=0.71, p=0.07\right.$. Figure $\left.4 B\right)$, indicating that gatekeeper conservation is reinforced with increasing aggregation propensity of the APR to which it is associated. Importantly, we find that not only the amino acid identity of gatekeepers is increasingly conserved $\left(\mathrm{R}^{2}=0.89, \mathrm{p}=0.015\right)$ but also that codon conservation of gatekeepers increases $\left(\mathrm{R}^{2}=0.93, \mathrm{p}=0.007\right)$ with increasing aggregation propensity of APRs (Figure 4C). 


\section{Discussion}

Our work demonstrates that, as a result of negative selection, gatekeepers are mutational cold spots in the E.coli genome and that the mutation rate of gatekeepers is coupled to the aggregation propensity of the APR with which they are associated. In addition we find that gatekeepers destabilize protein structure. Together these findings suggest that the enrichment of charged residues and prolines at the flanks of APRs is a selective process and that gatekeepers constitute a true functional class dedicated to the control of protein aggregation. Indeed, not only do gatekeepers destabilize protein structure. Strikingly they also slow down protein folding ${ }^{30}$ resulting in a folding process that is less aggregation-prone and thus more efficient, supporting the view that gatekeeper function results from negative selection against aggregation.

The presence of gatekeepers at the flanks of APRs has important consequences for protein structure. As gatekeepers are generally buried their charged moieties are almost systematically accommodated by H-bonds or salt-bridges. In fact $60 \%$ of highly buried gatekeepers (side chain burial $>60 \%$ ) form salt-bridges while the rest is involved in H-bonds with polar residues or main chain atoms. As gatekeepers are present in every E. coli protein they are at the origin of at least $15 \%$ of all salt-bridges in E. coli.

Beyond their direct effects at the protein structural level, evidence suggests that gatekeepers might have been co-opted by the proteostatic machinery of the cell. Chaperone binding experiments showed that gatekeeper residues contribute to chaperone binding selectivity by favouring binding to positively charged residues and prolines $4 ; 8$, whereas negatively charged gatekeepers appear to be more effective structural breakers ${ }^{31}$.

The interplay of gatekeepers with the proteostatic machinery remains to be further investigated. Interestingly however, in E. coli gatekeeper identity affects protein abundance by modulating protein synthesis $\&$ degradation, but also the toxicity of misfolded proteins by modulating inclusion body formation ${ }^{18}$.

Finally, gatekeeper identity is not only conserved at the amino acid level but also at the codon level. It remains to be shown whether the conservation of synonymous sites can be explained by sequence composition or whether it is the result of selection, which could indicate a co-translational role for gatekeepers in interaction with the ribosome ${ }^{32 ; 33}$. 


\section{Materials and Methods}

Dataset

We used expression data and multiple sequence alignments for 2659 protein families in E. coli from Martincorena et al. As a reference we used the annotation of the reference strain K12 MG1655. As transmembrane proteins are not under selective pressure to minimize their aggregation tendency, we filtered these with TMHMM ${ }^{34}$. This resulted in a dataset of 1955 protein families.

\section{Determination of APRs and gatekeepers}

TANGO ${ }^{26}$ was used to determine aggregation-prone regions (APRs) in human proteins. This resulted in an aggregation propensity (0-100\%) for each residue, whereby an aggregating segment is defined as a continuous stretch of at least five consecutive residues, each with a TANGO score higher than 5\%. As each APR has an average TANGO score, ranging from 0 to 100, aggregating segments are binned based on this average value.

The gatekeeping flanks are the positions before and after an aggregation-prone region, with each $\mathrm{P}, \mathrm{R}, \mathrm{K}, \mathrm{D}$ or $\mathrm{E}$ counting as gatekeeper. No distinction was made between the $\mathrm{N}$ or $\mathrm{C}$ terminus of the aggregating stretch.

\section{Structural modeling}

Crystal structures were selected according to the following criteria: (1) at least $60 \%$ sequence identity with the sequence of interest and (2) a resolution of at least $4.0 \AA$. All modeling was performed using the FoldX $3 \mathrm{~b} 5.1$ force field ${ }^{28}$ and tool suite. In total, this resulted in a set of 797 protein families having structural information, consisting of 436 structures and 361 homology models.

The contribution to stability and steric burial for each residue was obtained using the SequenceDetail command in FoldX 3b5.1. The PrintNetworks command was used to retrieve the H-bonding partners. Saltbridge information was extracted from the 797 models with YASARA ${ }^{35}$. A saltbridge was detected if two opposite charged residues had at least one atom-atom contact closer than 4.5 Angstrom. Such a contact must also be formed between the hydrogen bonding-capable sidechain atoms of both residues.

$\underline{\text { Sequence analysis }}$ 
Based on the mutiple sequence alignments, each codon was classified as either a) conserved in all strains, b) codon with synonymous SNP, c) codon with nonsynonymous SNP or d) codon with gaps or ambiguous nucleotides. The latter were excluded from this analysis. Using this assignment, the average genome conservation was calculated.

As the average genome conservation is high, we calculated the deviation from this genome average. The average conservation for residues grouped by their structural feature $(\mathrm{Y})$ was corrected by the average genome conservation $(\mathrm{X})$. The percentage deviation was calculated as follows: $(\mathrm{Y}-\mathrm{X}) / \mathrm{X} * 100$. 


\section{Acknowledgements}

We thank Inigo Martincorena and Nicholas Luscombe for sharing the multiple sequence alignements that form the basis of our analysis and providing critical comments on the manuscript. The Switch Laboratory was supported by grants from VIB, University of Leuven, the Funds for Scientific Research Flanders (FWO), the Flanders Institute for Science and Technology (IWT) and the Federal Office for Scientific Affairs of Belgium (Belspo), IUAP P7/16. GDB held a personal grant from IWT. 


\section{List of figures}

Figure 1: Aggregation analysis of the reference strain K12 MG1655. A: Difference plot of amino acid composition at the flank of APRs compared with average proteome composition. Values above or below 0 denote an increase or decrease in frequency, respectively. B: Histogram showing the TANGO score distribution of APRs in the proteome. A higher TANGO score indicates a higher aggregation propensity.

Figure 2: Structural information of the APRs. A-C: Notched boxplot for the sideand main chain burial, and $\mathrm{dG}$ for the residues constituting the APR, by the TANGO score interval of the surrounding APR. It is assumed that a higher TANGO score indicates a higher aggregation propensity.

Note on the boxplot: The box shows the interquartile range of the 25 to 75 percentile. The line in the middle of the box indicates the median and the notch in the box indicates the $95 \%$ confidence interval around the median. The lines extending from the box indicate the $99 \%$ limits of the distribution. Outliers are shown as circles.

Figure 3: Structural information of the surrounding gatekeepers. A-C: Notched boxplot for the side- and main chain burial, and dG for the gatekeepers surrounding the APR, by the TANGO-score interval of this APR. D-E: Percentage of gatekeepers forming a salt bridge or H-bond, respectively, by side chain burial intervals of the gatekeeper.

Figure 4: Conservation of APRs and gatekeepers. Plots A and B show the percentage deviation from the average genome-wide rate of non-synonymous mutations. A) For all residues within APRs, averaged per TANGO score interval of those APRs and B) For all gatekeeper residues averaged by the TANGO score interval of the surrounding APR. C) Deviation from the average conservation and synonymous mutation rate for gatekeepers averaged by the TANGO score interval of the surrounding APR. 


\section{References}

1. Chiti, F., Taddei, N., Baroni, F., Capanni, C., Stefani, M., Ramponi, G. \& Dobson, C. M. (2002). Kinetic partitioning of protein folding and aggregation. Nat Struct Biol 9, 137-43.

2. Esteras-Chopo, A., Serrano, L. \& de la Paz, M. L. (2005). The amyloid stretch hypothesis: Recruiting proteins toward the dark side. Proceedings of the National Academy of Sciences of the United States of America 102, 16672-16677.

3. Rousseau, F., Schymkowitz, J. \& Serrano, L. (2006). Protein aggregation and amyloidosis: confusion of the kinds? Current Opinion in Structural Biology 16, 118-126.

4. Rousseau, F., Serrano, L. \& Schymkowitz, J. W. H. (2006). How evolutionary pressure against protein aggregation shaped chaperone specificity. Journal of Molecular Biology 355, 1037-1047.

5. Monsellier, E. \& Chiti, F. (2007). Prevention of amyloid-like aggregation as a driving force of protein evolution. EMBO Rep 8, 737-42.

6. Monsellier, E., Ramazzotti, M., de Laureto, P. P., Tartaglia, G. G., Taddei, N., Fontana, A., Vendruscolo, M. \& Chiti, F. (2007). The distribution of residues in a polypeptide sequence is a determinant of aggregation optimized by evolution. Biophysical Journal 93, 4382-4391.

7. de Groot, N. S. \& Ventura, S. (2010). Protein aggregation profile of the bacterial cytosol. PLoS One 5, e9383.

8. Reumers, J., Maurer-Stroh, S., Schymkowitz, J. \& Rousseau, F. (2009). Protein Sequences Encode Safeguards Against Aggregation. Human Mutation 30, 431-437.

9. Buell, A. K., Tartaglia, G. G., Birkett, N. R., Waudby, C. A., Vendruscolo, M., Salvatella, X., Welland, M. E., Dobson, C. M. \& Knowles, T. P. J. (2009). Position-Dependent Electrostatic Protection against Protein Aggregation. Chembiochem 10, 1309-1312.

10. De Baets, G., Reumers, J., Delgado Blanco, J., Dopazo, J., Schymkowitz, J. \& Rousseau, F. (2011). An Evolutionary Trade-Off between Protein Turnover Rate and Protein Aggregation Favors a Higher Aggregation Propensity in Fast Degrading Proteins. PLoS Comput Biol 7, e1002090.

11. Parrini, C., Taddei, N., Ramazzotti, M., Degl'Innocenti, D., Ramponi, G., Dobson, C. M. \& Chiti, F. (2005). Glycine residues appear to be evolutionarily conserved for their ability to inhibit aggregation. Structure (Camb) 13, 1143-51.

12. Gerling, U. I., Brandenburg, E., von Berlepsch, H., Pagel, K. \& Koksch, B. (2011). Structure analysis of an amyloid-forming model peptide by a systematic glycine and proline scan. Biomacromolecules 12, 2988-96.

13. Sievers, S. A., Karanicolas, J., Chang, H. W., Zhao, A., Jiang, L., Zirafi, O., Stevens, J. T., Munch, J., Baker, D. \& Eisenberg, D. (2011). Structure-based design of non-natural amino-acid inhibitors of amyloid fibril formation. Nature 475, 96-100. 
14. Erbse, A., Schmidt, R., Bornemann, T., Schneider-Mergener, J., Mogk, A., Zahn, R., Dougan, D. A. \& Bukau, B. (2006). ClpS is an essential component of the N-end rule pathway in Escherichia coli. Nature 439, 753-6.

15. Schlieker, C., Weibezahn, J., Patzelt, H., Tessarz, P., Strub, C., Zeth, K., Erbse, A., Schneider-Mergener, J., Chin, J. W., Schultz, P. G., Bukau, B. \& Mogk, A. (2004). Substrate recognition by the AAA+ chaperone ClpB. Nat Struct Mol Biol 11, 607-15.

16. Patzelt, H., Rudiger, S., Brehmer, D., Kramer, G., Vorderwulbecke, S., Schaffitzel, E., Waitz, A., Hesterkamp, T., Dong, L., Schneider-Mergener, J., Bukau, B. \& Deuerling, E. (2001). Binding specificity of Escherichia coli trigger factor. Proc Natl Acad Sci U S A 98, 14244-9.

17. Rudiger, S., Germeroth, L., SchneiderMergener, J. \& Bukau, B. (1997). Substrate specificity of the DnaK chaperone determined by screening cellulose-bound peptide libraries. Embo Journal 16, 1501-1507.

18. Beerten, J., Jonckheere, W., Rudyak, S., Xu, J., Wilkinson, H., De Smet, F., Schymkowitz, J. \& Rousseau, F. (2012). Aggregation gatekeepers modulate protein homeostasis of aggregating sequences and affect bacterial fitness. Protein Eng Des Sel 25, 357-66.

19. Tartaglia, G. G. \& Caflisch, A. (2007). Computational analysis of the S. cerevisiae proteome reveals the function and cellular localization of the least and most amyloidogenic proteins. Proteins 68, 273-8.

20. Chen, Y. W. \& Dokholyan, N. V. (2008). Natural selection against protein aggregation on self-interacting and essential proteins in yeast, fly, and worm. Molecular Biology and Evolution 25, 1530-1533.

21. Tartaglia, G. G. \& Vendruscolo, M. (2010). Proteome-Level Interplay between Folding and Aggregation Propensities of Proteins. Journal of Molecular Biology 402, 919-928.

22. Ota, M., Kinoshita, K. \& Nishikawa, K. (2003). Prediction of catalytic residues in enzymes based on known tertiary structure, stability profile, and sequence conservation. J Mol Biol 327, 1053-64.

23. Meiering, E. M., Serrano, L. \& Fersht, A. R. (1992). Effect of active site residues in barnase on activity and stability. J Mol Biol 225, 585-9.

24. Shoichet, B. K., Baase, W. A., Kuroki, R. \& Matthews, B. W. (1995). A relationship between protein stability and protein function. Proc Natl Acad Sci U S A 92, 452-6.

25. Martincorena, I., Seshasayee, A. S. \& Luscombe, N. M. (2012). Evidence of non-random mutation rates suggests an evolutionary risk management strategy. Nature 485, 95-8.

26. Fernandez-Escamilla, A. M., Rousseau, F., Schymkowitz, J. \& Serrano, L. (2004). Prediction of sequence-dependent and mutational effects on the aggregation of peptides and proteins. Nat Biotechnol 22, 1302-6.

27. Westbrook, J., Feng, Z. K., Chen, L., Yang, H. W. \& Berman, H. M. (2003). The Protein Data Bank and structural genomics. Nucleic Acids Research 31, 489-491.

28. Schymkowitz, J., Borg, J., Stricher, F., Nys, R., Rousseau, F. \& Serrano, L. (2005). The FoldX web server: an online force field. Nucleic Acids Res 33, W382-8.

29. Kryazhimskiy, S. \& Plotkin, J. B. (2008). The population genetics of dN/dS. PLoS Genet 4, e1000304. 
30. Kurnik, M., Hedberg, L., Danielsson, J. \& Oliveberg, M. (2012). Folding without charges. Proceedings of the National Academy of Sciences 109, 5705-5710.

31. Jespers, L., Schon, O., Famm, K. \& Winter, G. (2004). Aggregation-resistant domain antibodies selected on phage by heat denaturation. Nat Biotechnol 22, 1161-5.

32. Willmund, F., del Alamo, M., Pechmann, S., Chen, T., Albanese, V., Dammer, E. B., Peng, J. \& Frydman, J. (2013). The cotranslational function of ribosome-associated Hsp70 in eukaryotic protein homeostasis. Cell 152, 196-209.

33. Komar, A. A., Lesnik, T. \& Reiss, C. (1999). Synonymous codon substitutions affect ribosome traffic and protein folding during in vitro translation. FEBS Lett 462, 387-91.

34. Krogh, A., Larsson, B., von Heijne, G. \& Sonnhammer, E. L. (2001). Predicting transmembrane protein topology with a hidden Markov model: application to complete genomes. J Mol Biol 305, 567-80.

35. Krieger, E., Koraimann, G. \& Vriend, G. (2002). Increasing the precision of comparative models with YASARA NOVA--a self-parameterizing force field. Proteins 47, 393-402. 



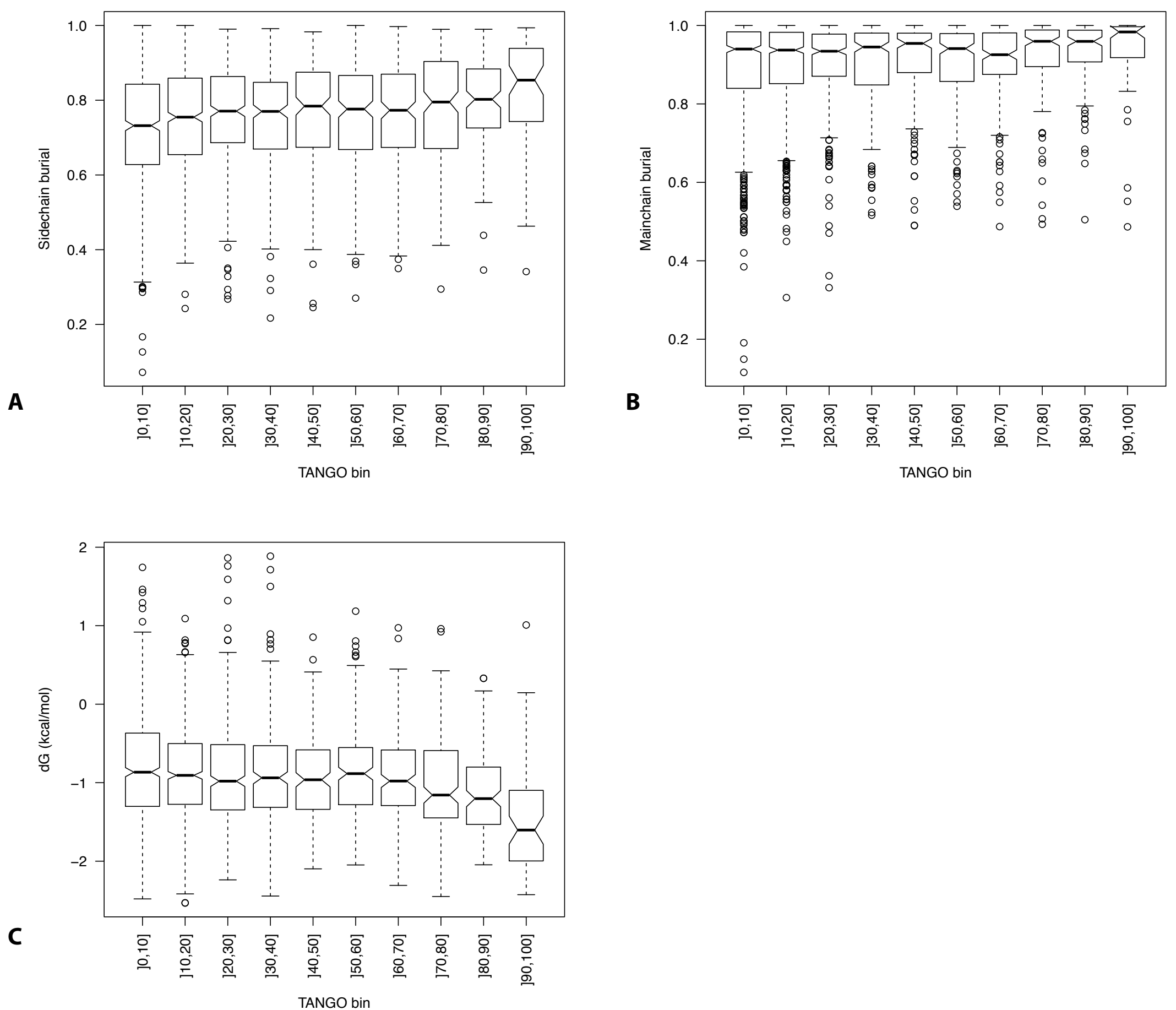

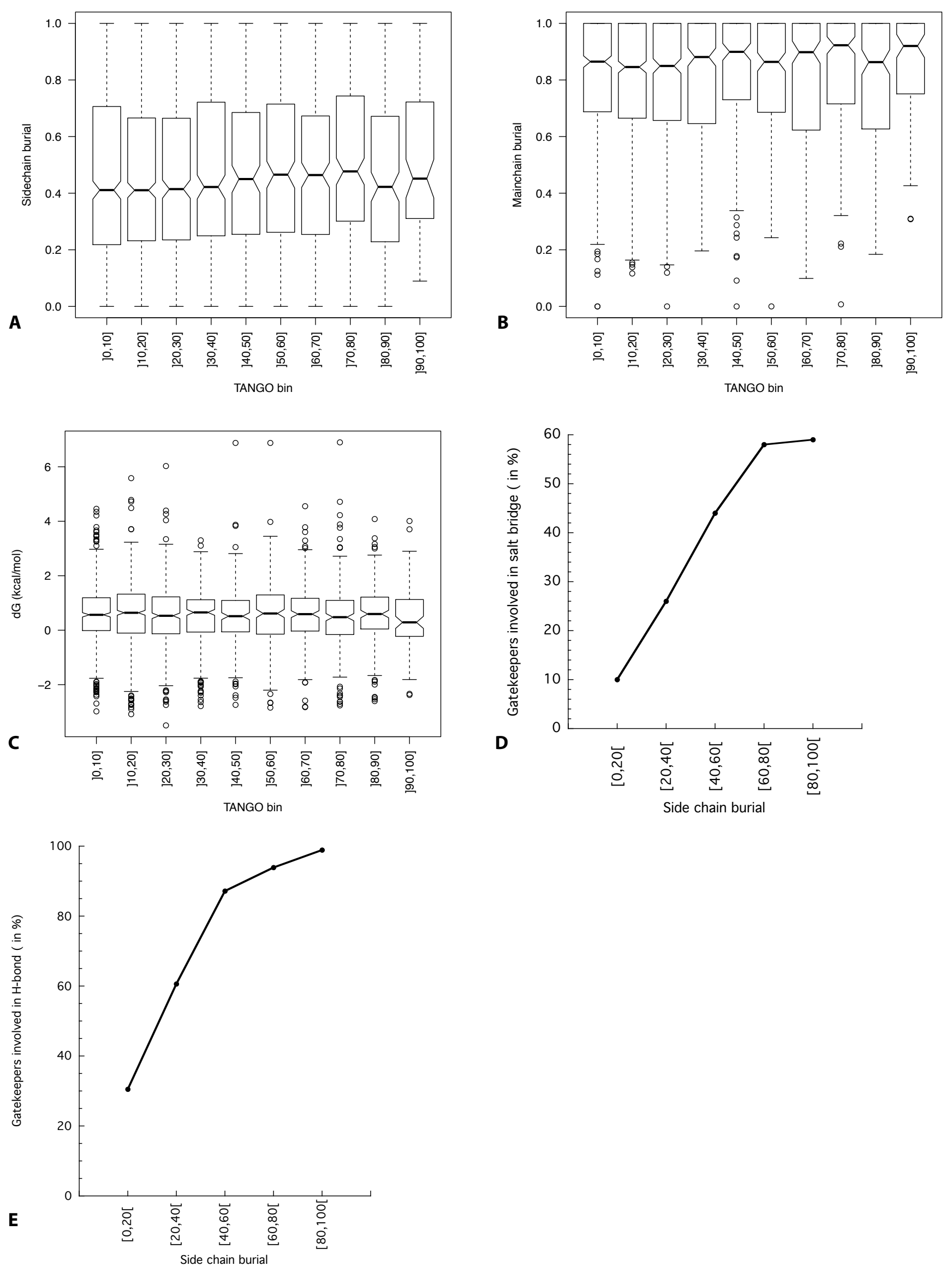

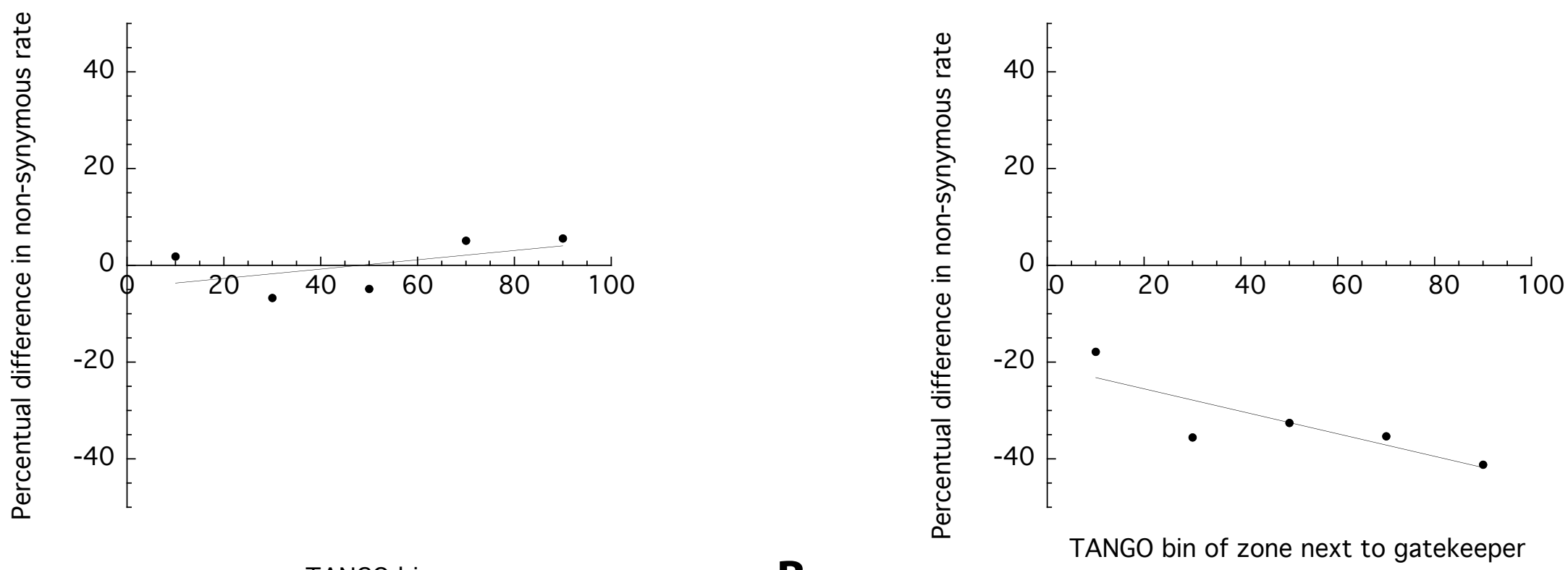

A

TANGO bin

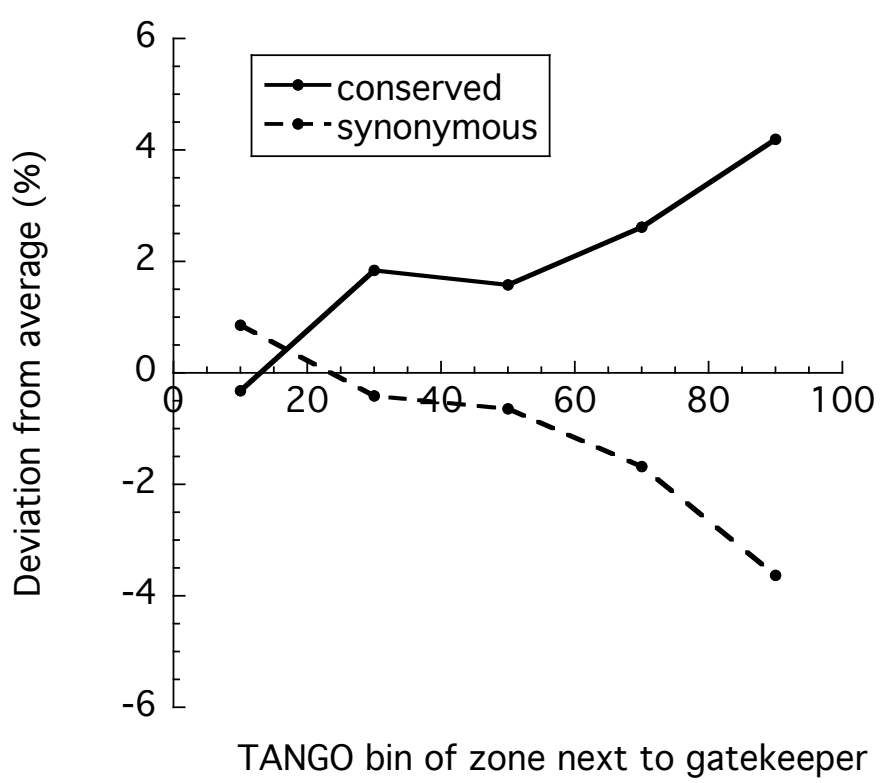

C 